\title{
Outcome of Colorectal Cancer Patients Treated with Combination Bevacizumab Therapy: A Pooled Retrospective Analysis of Three European Cohorts from the Angiopredict Initiative
}

\author{
Johannes Betge ${ }^{a, d}$ Ana Barat ${ }^{f}$ Verena Murphy ${ }^{g}$ Thomas Hielscher $^{e}$ \\ Nicole C. Van Grieken ${ }^{j}$ Sebastian Belle ${ }^{a}$ Tianzuo Zhan ${ }^{a, d}$ Nicolai Härtel $^{a}$ \\ Melanie Kripp ${ }^{b}$ Orna Bacon $^{f}$ Martijn Cordes $^{j}$ Elaine W. Kay ${ }^{h}$ \\ Henk M.W. Verheulk Maarten Neerincx ${ }^{k}$ Bryan Hennessy ${ }^{i} \quad$ Ralf D. Hofheinz ${ }^{b}$ \\ Timo Gaiser $^{c}$ Bauke Ylstraj Jochen H.M. Prehn ${ }^{f}$ Diether Lambrechts ${ }^{\prime}$ \\ Annette T. Byrne ${ }^{f}$ Matthias P. Ebert ${ }^{a}$ Nadine Schulte $^{a}$

\begin{abstract}
${ }^{a}$ Department of Medicine II, ${ }^{b}$ Interdisziplinäres Tumorzentrum, and ${ }^{\mathrm{C}}$ Institute of Pathology, University Hospital Mannheim, Medical Faculty Mannheim, Heidelberg University, Mannheim, d German Cancer Research Center (DKFZ), Division Signaling and Functional Genomics, and ' German Cancer Research Center (DKFZ), Division of Biostatistics, Heidelberg, Germany; ${ }^{\mathrm{f} C e n t r e ~ f o r ~ S y s t e m s ~ M e d i c i n e ~ a n d ~ D e p a r t m e n t ~ o f ~ P h y s i o l o g y ~ a n d ~ M e d i c a l ~ P h y s i c s, ~ R o y a l ~ C o l l e g e ~}$ of Surgeons in Ireland, 9/rish Clinical Oncology Research Group, ${ }^{\mathrm{h}}$ Department of Pathology, Beaumont Hospital, and 'Department of Medical Oncology, Beaumont Hospital, Dublin, Ireland; 'Department of Pathology, and kDepartment of Medical Oncology, VU University Medical Center, Amsterdam, The Netherlands; 'VIB Vesalius Research Center, KU Leuven, Leuven, Belgium
\end{abstract}

\section{Key Words}

Bevacizumab · Colorectal cancer · Combination

chemotherapy · Elderly patients · Prognostic factor

\begin{abstract}
Background/Aims: This study is aimed at analyzing the survival rates and prognostic factors of stage IV colorectal cancer patients from 3 European cohorts undergoing combination chemotherapy with bevacizumab. Methods: Progression free-survival (PFS) and overall survival (OS) were analyzed in 172 patients using the Kaplan-Meier method and uni- and multivariable Cox proportional hazards regression models. Results: The median PFS was 9.7 and the me-
\end{abstract}

\section{KARGER}

E-Mail karger@karger.com www.karger.com/dig dian OS 27.4 months. Patients treated at centers in Germany ( $n=97)$, Ireland $(n=32)$, and The Netherlands $(n=43)$ showed a median PFS of 9.9, 9.2, and 9.7 months, OS of 34.0, 20.5 , and 25.1 months, respectively. Patients $>65$ years had a significantly shorter PFS (9.5 vs. 9.8 months) but not OS (27.4 vs. 27.5 months) than younger patients. High tumor grade (G3/4) was associated with a shorter PFS, T4 classification with both shorter PFS and OS. Fluoropyrimidine (FP) chemotherapy backbones (doublets and single) had comparable outcomes, while patients not receiving FP backbones had a shorter PFS. In multivariable analysis, age and non-FP

\section{A.B., V.M., T.H. and N.C.V.G. are joint second authors.}

(C) 2016 S. Karger AG, Basel

0012-2823/16/0943-0129\$39.50/0
Prof. Dr. Matthias P. Ebert and Dr. Johannes Betge

Department of Medicine II, University Hospital Mannheim, Medical Faculty Mannheim Heidelberg University, Theodor-Kutzer-Ufer 1-3, DE-68167 Mannheim (Germany)

E-Mail matthias.ebert@medma.uni-heidelberg.de and

johannes.betge@medma.uni-heidelberg.de 
backbone were associated with inferior PFS, T4 classification and therapy line $>2$ nd were significantly associated with poor PFS and OS. Conclusion: The observed survival rates confirm previous studies and demonstrate reproducible benefits of combination bevacizumab regimens. Classification T4, non-FP chemotherapy backbone, and age $>65$ were associated with inferior outcome.

(c) 2016 S. Karger AG, Basel

\section{Introduction}

Colorectal cancer (CRC) is globally a major cause of cancer morbidity and mortality, being the third most commonly diagnosed cancer in males and the second most common in females with more than 1.4 million new cancer cases diagnosed in 2012 [1]. Approximately, one third of patients initially present with metastases and almost $50 \%$ of patients diagnosed at early stage will eventually develop metastatic or irresectable locally advanced disease [2].

The treatment of metastatic or irresectable CRC has since the late 1950s been based on fluoropyrimidine (FP) 5-fluorouracil (5-FU), a nucleoside analog combined with calcium-folinate (leucovorin), leading to median survival rates of around 12 months [3-5]. Outcome benefits have been improved by adding oxaliplatin (e.g. FOLFOX) [6] or irinotecan (e.g. FOLFIRI) [7], and monoclonal antibodies (cetuximab, panitumumab, bevacizumab), fusion proteins (aflibercept), and tyrosine kinase inhibitors (regorafenib), leading to median overall survival (OS) rates of around 2 years $[8,9]$. Bevacizumab is a humanized monoclonal antibody against vascular endothelial growth factor, one of the major growth factors involved in vessel formation, which is crucial for the growth and invasion of malignant cells [10]. Randomized clinical trials have revealed a significant benefit in progression-free-survival (PFS) and OS, when bevacizumab is added to 5-FU based chemotherapy regimens [11-19].

However, only a subset of patients respond to targeted agents such as bevacizumab, and the overall clinical benefit is limited. Also, toxic side effects and high treatment costs should be considered [20]. Therefore, new methods to stratify patients before treatment with bevacizumab, based on predictive- and prognostic factors are urgently needed. Angiopredict (www.angiopredict.com) is a Framework Programme 7 (FP7) European Commission funded, multidisciplinary and multi-institutional research project that seeks to identify predictive genomic biomarker signatures for metastatic CRC patients receiv- ing combination bevacizumab therapy. Multi-omic molecular analyses are currently being performed on retrospectively retrieved samples from patients undergoing chemotherapy with or without bevacizumab therapy, collected at centers in Ireland, the Netherlands, and Germany. Validation of findings will be performed on tissues prospectively collected in the ongoing AC-Angiopredict Phase II Exploratory trial (NCT01822444).

Herein, we sought to evaluate the clinical and pathological characteristics, the survival rates, and clinical prognostic factors in a pooled retrospective cohort of patients from the Angiopredict study that have been treated with combination bevacizumab therapy.

\section{Patients and Methods}

\section{Patients}

Patients with advanced (locally irresectable or metastatic) CRC commencing combination chemotherapy including Bevacizumab between July 2004 and April 2012 were included in this analysis. Clinicopathological data were collected within 3 different cohorts: (1) University Hospital Mannheim, Heidelberg University, Mannheim, Germany (UHEI); (2) VU University Medical Center Amsterdam, the Netherlands and several other Dutch hospitals (VUMC); and (3) Royal College of Surgeons in Ireland, Beaumont Hospital, Dublin, Ireland (RCSI). Criteria for inclusion were: (1) histologically proven diagnosis of colon or rectum adenocarcinoma, either metastasized or locally advanced and irresectable, (2) combination chemotherapy with a regimen including bevacizum$\mathrm{ab}$ at any line of chemotherapy.

$\mathrm{T}$ - and N-classifications, grading, and localization of the tumor samples were collected by reviewing patients' records. T- and Nclassifications, and grading were routinely evaluated by different pathologists from the participating centers using AJCC/UICC and WHO guidelines, respectively $[21,22]$.

Colon cancers located from caecum to hepatic flexure were defined as right-sided cancers, and tumors located from transverse colon to sigmoid as left-sided. KRAS mutations in codons $12 / 13$ were assessed.

\section{Chemotherapy}

Chemotherapy, administered together with Bevacizumab, included the following regimens: (A) Fluoropyrimidine (FP)-based chemotherapy (5-fluorouracil (5-FU), 5-FU and leucovorin, capecitabine), (B) oxaliplatin doublets (FOLFOX, CapOX/XelOX, oxaliplatin and raltitrexed), (C) irinotecan doublets (FOLFIRI, CapIRI/XelIRI, irinotecan and raltitrexed) and (D) others (irinotecan, oxaliplatin, mitomycin or no backbone). All patients from the VUMC cohort were treated with either FOLFOX or CapOX and bevacizumab.

\section{Follow-Up}

The observation period for each patient commenced with the initiation of bevacizumab treatment. Clinical data for the 3 cohorts were routinely collected and documented by the treating physician. The follow-up period for the UHEI, VUMC and RSCI co- 
horts started on July 28, 2004, September 7, 2004, and August 18, 2004, respectively. They ended on December 15, 2014, July 03, 2013, and June 02, 2015, respectively. Follow-up included CT scans or abdominal ultrasound and chest X-ray every 3 months.

Institutional Review Board approval was obtained from the responsible Ethics Committees for all participating study centers.

\section{Statistical Analysis}

The data from the 3 cohorts were assessed via pooled analysis using individual patient data. Fisher's exact test was used to compare the distribution of clinicopathological factors between centers. PFS was defined as the time from start of bevacizumab therapy to progressive disease or death from any cause, whichever occurred first. Patients stopping bevacizumab therapy due to reasons other than progression or death were censored as of the date of treatment cessation. OS was defined as the time from start of bevacizumab to death from any cause. All patient data were administratively censored after 60 months.

Time to progression and death were retrospectively determined by chart review. PFS and OSs were investigated using the Kaplan-Meier method and compared by log-rank test or using Cox proportional hazards regression model. Log-rank tests and Cox regression were calculated with stratification by cohort to adjust for center effect.

For multivariable Cox proportional hazards regression models, multiple imputation $(B=100)$ of missing values was performed using the predictive mean matching algorithm as implemented in $\mathrm{R}$ package Hmisc [23].

All statistical calculations were performed using R (version 3.1, R Foundation for Statistical Computing, Vienna, Austria. http:// www.R-project.org/). All reported p values were 2 -sided with significance at $\mathrm{p}<0.05$.

\section{Results}

\section{Patient Characteristics}

A total of 172 patients were included in this analysis. Of which, 97 patients were treated at UHEI, 32 at the RCSI, and 43 were part of the VUMC cohort.

Fifty-four (31\%) patients were females and 118 (69\%) males, $115(67 \%)$ had a primary colon and $57(33 \%)$ a primary rectum cancer. The median age at the start of treatment with bevacizumab was 65 years (mean 63.7, range $27-84$ ). Eighty patients (47\%) were older than 65 years, 90 patients $(53 \%)$ were 65 years or younger. One hundred twenty nine patients (75\%) were treated with a chemotherapy regimen that included bevacizumab in first line, 33 (19\%) in second line, 10 (6\%) in third or later lines. Fiftyeight patients $(34 \%)$ received an irinotecan doublet chemotherapy backbone, $83(48 \%)$ an oxaliplatin doublet. Twenty-four patients (14\%) received bevacizumab together with a FP monotherapy, 7 patients (4\%) were treated with bevacizumab and irinotecan or with bevacizumab as monotherapy. Other patient characteristics are shown in table 1 . Tumor grade was available for 146 patients, T classification for 160, N classification for 156 patients. Data regarding surgery of the primary tumor or of metastases could not be determined for all patients and could therefore not be analyzed for the pooled retrospective cohort. The 3 cohorts included differed significantly with respect to gender, age, T-classification, grading, location of the primary tumor, treatment line, and chemotherapy backbone.

The median follow-up time was 48.1 months (95\% CI 40.5-56.2). The median follow-up times of the UHEI, RCSI, and VUMC cohorts were 30.9, 60.0, and 60.0 months, respectively. At the end of follow-up, 121 patients (70\%) experienced disease progression, 104 (60\%) were deceased, while 68 patients $(40 \%)$ were censored with respect to OS.

\section{Survival Analysis}

Patients with CRC that underwent combination therapy with bevacizumab had a median PFS of 9.67 months (95\% CI 9.18-10.56) and a median OS of 27.4 months (95\% CI 22.9-32.7; fig. 1a, b). Patients from UHEI, RCSI, and VUMC cohorts had a median PFS of 9.9, 9.2, and 9.7 months, respectively. The OS of patients from UHEI, RCSI, and VUMC were 34.0, 20.5, and 25.1 months, respectively.

Patients who were older than 65 years at the start of treatment with bevacizumab had a significantly shorter PFS (9.5 vs. 9.8 months, $\mathrm{p}=0.01$ ), but no significant difference in the OS was observed ( 27.4 vs. 27.5 months, $\mathrm{p}=$ 0.43 ) in patients following treatment with bevacizumab (fig. 2a, b). Regarding gender, no statistically significant difference in the median PFS (females: 10 months vs. males: 9.5 months, $\mathrm{p}=0.92$ ) or median OS (females: 25.2 months, vs. males: 27.5 months, $\mathrm{p}=0.34$ ) was observed.

Higher tumor grades (G3-4 vs. G1-2) correlated with a shorter PFS (7 vs. 9.9 months, $\mathrm{p}=0.033$ ), but had no impact on OS ( $\mathrm{p}=0.77)$. Tumor localization (right-sided colon cancer, left-sided colon cancer, rectal cancer) had no significant influence on PFS of patients on bevacizumab treatment (right-sided 9.2 months, left-sided 9.3 months, rectum 10.4 months, $\mathrm{p}=0.69$ ). There was a trend towards worse OS of patients with right-sided colon cancer compared to patients with left sided cancers and rectal cancers $(19.1,31.9$, and 25 months, respectively, $\mathrm{p}=0.24$ ). The presence of lymph node metastases had no significant impact on the outcome regarding both PFS (N0: 10 months, N1-2: 9.7 months, $\mathrm{p}=0.58$ ) and OS (N0: 28.3 months, N1-2: 27.4 months, $\mathrm{p}=0.64)$ in this setting of predominantly metastasized cancers. Of note, $\mathrm{N}$-classification had an age-dependent impact on PFS (interaction 
Table 1. Characteristics of patients treated with bevacizumab

\begin{tabular}{|c|c|c|c|c|c|c|c|c|c|}
\hline & \multicolumn{2}{|c|}{ UHEI } & \multicolumn{2}{|c|}{ RCSI } & \multicolumn{2}{|c|}{ VUMC } & \multicolumn{2}{|l|}{ All } & \multirow[t]{2}{*}{$\mathrm{p}$ value } \\
\hline & $\mathrm{n}$ & $\%$ & $\mathrm{n}$ & $\%$ & $\mathrm{n}$ & $\%$ & $\mathrm{n}$ & $\%$ & \\
\hline Gender & & & & & & & & & $<0.001$ \\
\hline Female & 37 & 38 & 4 & 13 & 13 & 30 & 54 & 31 & \\
\hline Male & 60 & 62 & 28 & 88 & 30 & 70 & 118 & 69 & \\
\hline Age, years & & & & & & & & & 0.001 \\
\hline$>65$ & 57 & 59 & 12 & 40 & 11 & 26 & 80 & 47 & \\
\hline$\leq 65$ & 40 & 41 & 18 & 60 & 32 & 74 & 90 & 53 & \\
\hline T-classification & & & & & & & & & 0.005 \\
\hline 1 & 4 & 4 & 0 & 0 & 0 & 0 & 4 & 3 & \\
\hline 2 & 13 & 14 & 2 & 7 & 4 & 10 & 19 & 12 & \\
\hline 3 & 53 & 59 & 12 & 41 & 32 & 78 & 97 & 61 & \\
\hline 4 & 20 & 22 & 15 & 52 & 5 & 12 & 40 & 25 & \\
\hline N-classification & & & & & & & & & 0.49 \\
\hline Negative & 30 & 35 & 7 & 23 & 12 & 30 & 49 & 31 & \\
\hline Positive & 56 & 65 & 23 & 77 & 28 & 70 & 107 & 69 & \\
\hline Tumor grade & & & & & & & & & $<0.001$ \\
\hline Low (1-2) & 46 & 60 & 27 & 96 & 35 & 85 & 108 & 74 & \\
\hline High (3-4) & 31 & 40 & 1 & 4 & 6 & 14 & 38 & 26 & \\
\hline Localization & & & & & & & & & 0.038 \\
\hline Right-sided & 16 & 17 & 12 & 39 & 8 & 20 & 36 & 21 & \\
\hline Left-sided & 43 & 44 & 17 & 55 & 20 & 48 & 80 & 47 & \\
\hline Rectum & 38 & 39 & 2 & 6 & 14 & 33 & 54 & 32 & \\
\hline KRAS & & & & & & & & & 0.68 \\
\hline wt & 34 & 65 & 11 & 65 & 11 & 55 & 56 & 63 & \\
\hline mut & 18 & 35 & 6 & 35 & 9 & 45 & 33 & 37 & \\
\hline BVZ therapy line & & & & & & & & & $<0.001$ \\
\hline$>1$ & 60 & 62 & 26 & 81 & 43 & 100 & 129 & 75 & \\
\hline$>2$ & 29 & 30 & 4 & 13 & & & 33 & 19 & \\
\hline$\geq 3$ & 8 & 8 & 2 & 6 & & & 10 & 6 & \\
\hline CTX backbone & & & & & & & & & $<0.001$ \\
\hline FP single & 22 & 23 & 2 & 6 & & & 24 & 14 & \\
\hline IRI doublet & 51 & 53 & 7 & 22 & & & 58 & 34 & \\
\hline OX doublet & 21 & 22 & 19 & 59 & 43 & 100 & 83 & 48 & \\
\hline Other & 3 & 3 & 4 & 13 & & & 7 & 4 & \\
\hline Total & 97 & 100 & 32 & 100 & 43 & 100 & 172 & 100 & \\
\hline
\end{tabular}

$\mathrm{wt}=$ wild-type mut $=$ mutated IRI = irinotecan $\mathrm{OX}=$ oxaliplatin CTX = chemotherapy BVZ = bevacizumab.

$\mathrm{p}=0.003)$ : While N1-2 was of no prognostic impact in patients older than 65 years, younger patients $(\leq 65)$ with positive lymph node status had shorter PFS ( 8.8 months) than with negative lymph nodes (14 months, $\mathrm{p}=0.002)$. Tumor classification T4 (tumor penetration beyond visceral peritoneum or direct invasion into other organs or structures) at the time of diagnosis was significantly associated with both PFS (T1-T3: 10.3 months vs. T4: 6.8 months, $\mathrm{p}=0.004)$ and OS (T1-T3: 32 months vs. T4: 20.5 months, $\mathrm{p}<0.001)$. KRAS status could be obtained for 89 patients (52\%). No significant impact on PFS or OS was observed in our cohort (PFS: $\mathrm{p}=0.72$, OS: $\mathrm{p}=0.89$ ).
Regarding the chemotherapy backbone administered together with bevacizumab, significant differences in PFS were observed $(\mathrm{p}<0.001)$. These differences were, however, mainly attributable to 7 patients receiving bevacizumab with non-standard backbones that did not include a FP (irinotecan and bevacizumab; irinotecan, cetuximab and bevacizumab or bevacizumab monotherapy, PFS = 3.2 months), while all other backbones had similar PFS (irinotecan-doublets: 10.3 months, oxaliplatin-doublets: 9.7 months, FP-single: 10.4 months; fig 2c). No significant differences in OS were found regarding chemotherapy backbones ( $\mathrm{p}=0.58$; fig $2 \mathrm{~d}$ ). Patients receiving beva- 


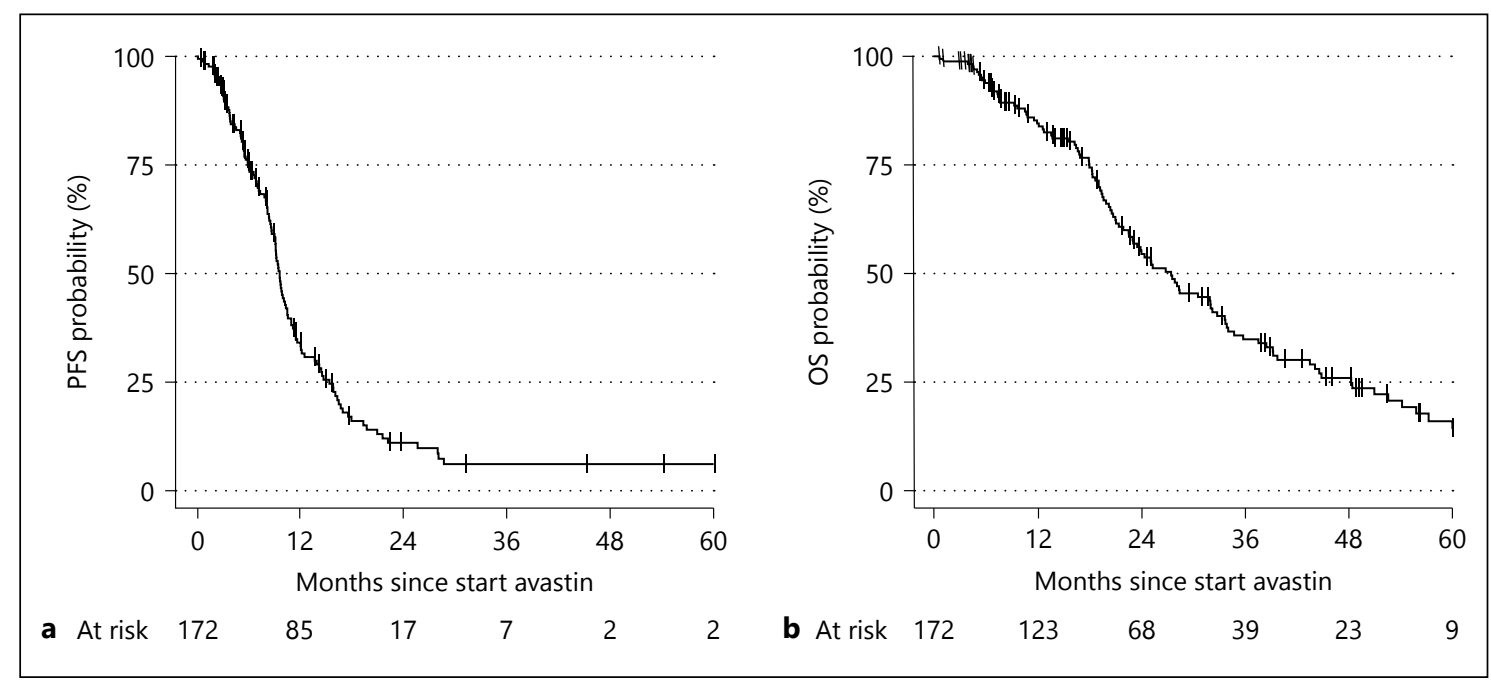

Fig. 1. PFS and OS of 172 CRC patients treated with bevacizumab. Kaplan-Meier analysis of (a) PFS and (b) OS of 172 patients treated with bevacizumab.

cizumab in first line $(\mathrm{n}=129)$, second line $(\mathrm{n}=33)$ or in third line or later lines $(\mathrm{n}=10)$ had a median PFS of 9.8, 9.9, and 5.5 months $(\mathrm{p}=0.06)$. The median OS of patients treated in first line, second line, or later lines were 27.4, 32.7, and 21.3 months $(\mathrm{p}=0.09)$, respectively.

Univariable Cox analyses of PFS and OS of all patients following treatment with bevacizumab are shown in table 2. In accordance with Kaplan-Meier analysis, age $>65$ years, higher tumor grade, T4 classification, treatment line $>2$ nd, and non-FP chemotherapy backbone were associated with a shorter PFS, while treatment line $>2$ nd and classification T4 predicted poor OS.

In a multivariable Cox proportional hazards regression model, a non-FP chemotherapy backbone and T4 classification were independently associated with adverse PFS. T4 classification and treatment line $>2$ nd were independently associated with adverse OS (table 3 ).

\section{Discussion}

Bevacizumab has emerged as an important component of palliative therapy in CRC. According to the data presented here, patients with CRC receiving bevacizum$a b$ in combination with chemotherapy had a median PFS of 9.8 months and a median OS of 23.7 months. PFS and OS in our cohorts are comparable with survival data from prospective clinical trials, in which a PFS of 7.3-10.3 months and an OS of 10.8-25.3 months have been observed [11, 13-15, 17, 19, 24-28].

Bevacizumab in CRC
The choice of chemotherapy (-backbone) regimen is important in the palliative treatment of CRC patients. To date, it has been mainly based on potential side effects, comorbidities, and preferences of patient and physician [29]. According to our findings, the chemotherapy backbone administered together with bevacizumab had no significant influence on either PFS or OS of patients, if a standard FPbased regimen was used. This is in accordance with previous studies, in which a median PFS of around 9-12 months and OS $>20$ months have been reported, regardless of chemotherapy (-doublet) used as backbone [29, 30]. Patients receiving backbones without a $\mathrm{FP}$ or without concomitant chemotherapy had significantly worse outcomes. It is possible that patients not receiving a FP did not receive a more debilitating chemotherapy due to co-morbidities, low performance status or frailty. Unfortunately, these factors could not be analyzed in this retrospective study. Nevertheless, it may be useful to discuss best supportive care with a patient in such a scenario, since the benefit of bevacizumab without chemotherapy or with non-standard backbones seems to be limited. Interestingly, patients treated with oxaliplatin or irinotecan doublets did not have longer PFS or OS than patients receiving only 5-FU or capecitabine together with bevacizumab. This may be explained by a larger fraction of elderly patients in our cohort that may benefit less from doublets and perform better with single 5-FU or capecitabine together with bevacizumab. However, since only about $15 \%$ of our patients were treated with $5-\mathrm{FU}$ or capecitabine and bevacizumab alone, the statistical power of our analysis is weakened due to the small sample size. 


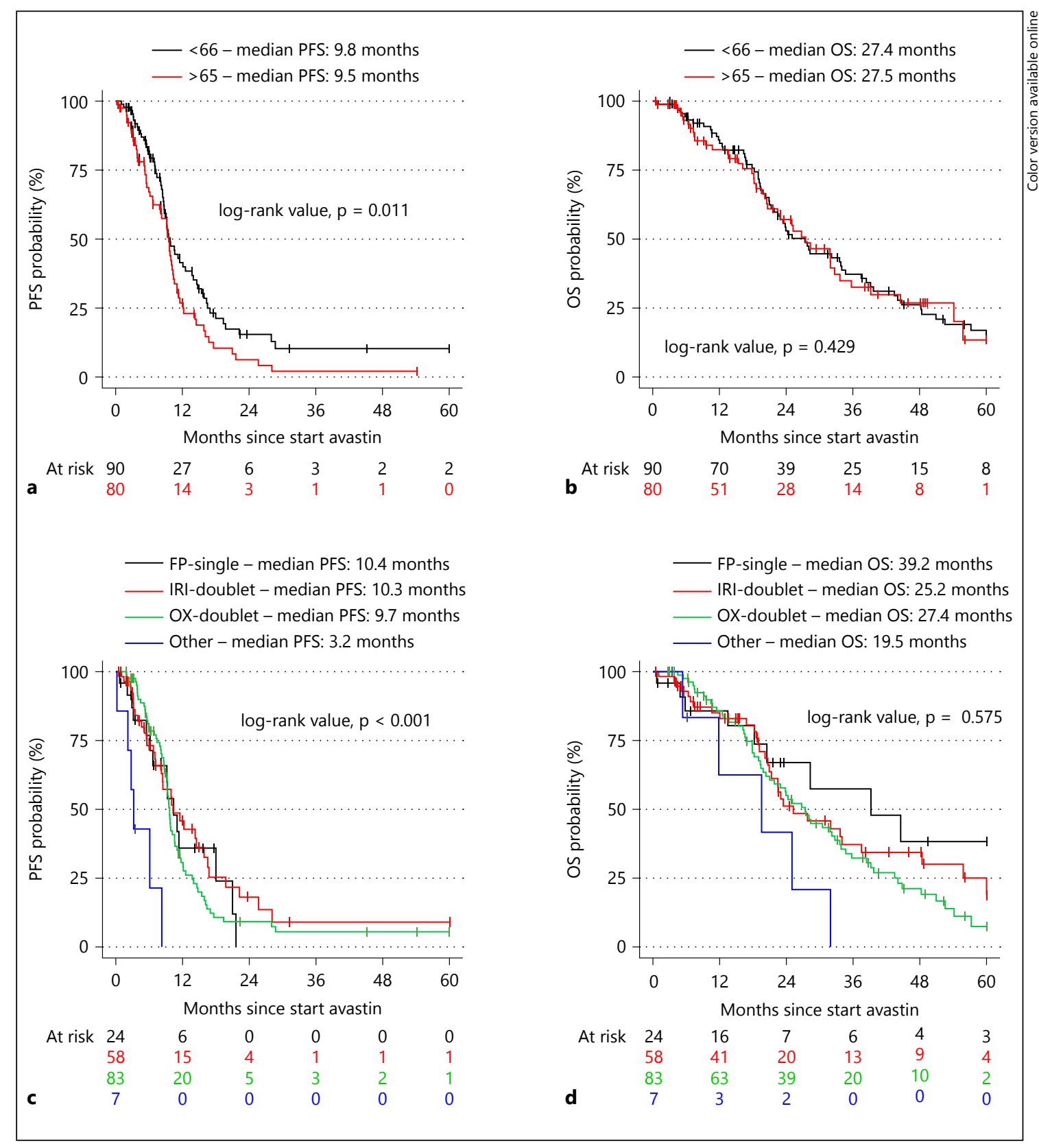

Fig. 2. Clinical prognostic factors influencing the survival of CRC patients treated with bevacizumab. Elderly patients $>65$ years had significantly shorter PFS (a) but not OS (b) than younger patients under chemotherapy with bevacizumab. PFS (c) and OS (d) are affected by the backbone chemotherapy regimen administered together with bevacizumab in Kaplan-Meier analysis due to shorter survival rates of patients receiving non-FP-backbones.
Regarding other clinicopathological factors, morphological grading of the primary tumor has an influence on PFS, but not on OS. Interestingly, even in the setting of patients with metastasized disease and mainly after resection of the primary tumor, T4 classification was significantly associated with worse PFS and OS and remained significantly associated with both PFS and OS in multivariable Cox analysis. This finding suggests that local tumor spread or recurrence may be a relevant, to date potentially underestimated prognostic determinant in metastasized CRC. Literature on the prognostic impact of pathologic variables of the primary tumor in 
Table 2. Univariable Cox analysis of prognostic factors under chemotherapy with bevacizumab

\begin{tabular}{|c|c|c|c|c|c|c|c|c|}
\hline \multirow[t]{2}{*}{ Variable } & \multirow[t]{2}{*}{ Level } & \multirow[t]{2}{*}{$\mathrm{n}$} & \multicolumn{3}{|l|}{ PFS } & \multicolumn{3}{|l|}{ OS } \\
\hline & & & HR & $95 \% \mathrm{CI}$ & $\mathrm{p}$ value & HR & $95 \% \mathrm{CI}$ & $\mathrm{p}$ value \\
\hline Gender & male vs. female & 172 & 1.02 & $0.68-1.53$ & 0.91 & 0.80 & $0.51-1.26$ & 0.35 \\
\hline Age & $>65$ vs. $\leq 65$ & 170 & 1.66 & $1.12-2.46$ & 0.011 & 1.19 & $0.77-1.82$ & 0.43 \\
\hline $\mathrm{T}^{\circ}$ & 4 vs. $1-3$ & 160 & 1.97 & $1.24-3.13$ & 0.004 & 2.21 & $1.37-3.57$ & 0.001 \\
\hline $\mathrm{N}$ & $1-2$ vs. 0 & 156 & 1.12 & $0.74-1.7$ & 0.59 & 1.11 & $0.71-1.73$ & 0.64 \\
\hline Grading & high vs. low & 146 & 1.74 & $1.04-2.9$ & 0.034 & 1.09 & $0.61-1.97$ & 0.77 \\
\hline \multirow{2}{*}{ Localization } & right vs. left & 170 & 1.13 & $0.70-1.82$ & 0.63 & 1.47 & $0.91-2.40$ & 0.12 \\
\hline & rectum vs. left & & 0.88 & $0.56-1.37$ & 0.56 & 0.97 & $0.60-1.58$ & 0.90 \\
\hline KRAS & mut vs. wt & 89 & 0.91 & $0.54-1.53$ & 0.71 & 1.04 & $0.59-1.82$ & 0.89 \\
\hline \multirow[t]{2}{*}{ Line } & 2 vs. 1 & 172 & 0.92 & $0.54-1.55$ & 0.74 & 1.28 & $0.71-2.31$ & 0.40 \\
\hline & $>2$ vs. 1 & & 2.71 & $1.11-6.59$ & 0.028 & 2.81 & $1.07-7.41$ & 0.037 \\
\hline \multirow[t]{3}{*}{ Backbone } & IRI doubl vs. FP & 172 & 0.90 & $0.48-1.68$ & 0.74 & 1.25 & $0.59-2.65$ & 0.57 \\
\hline & OX doubl vs. FP & & 1.12 & $0.56-2.24$ & 0.75 & 1.01 & $0.43-2.40$ & 0.97 \\
\hline & other vs. FP & & 8.62 & $2.83-26.19$ & $<0.001$ & 1.91 & $0.56-6.50$ & 0.30 \\
\hline
\end{tabular}

$\mathrm{n}=$ Number $\mathrm{HR}=$ hazard ratio; $\mathrm{p}=$ wald $\mathrm{p}$ value; $\mathrm{m}=$ male $\mathrm{f}=$ female; mut = mutated wt = wild-type; dou$\mathrm{bl}=$ doublet; $\mathrm{IRI}=$ irinotecan; $\mathrm{OX}=$ oxaliplatin.

Table 3. Multivariable Cox proportional hazards regression analysis of prognostic factors in 172 patients receiving chemotherapy with bevacizumab

\begin{tabular}{|c|c|c|c|c|c|c|c|}
\hline \multirow[t]{2}{*}{ Variable } & \multirow[t]{2}{*}{ Level } & \multicolumn{3}{|l|}{ PFS } & \multicolumn{3}{|l|}{ OS } \\
\hline & & HR & $95 \%$ CI & $\mathrm{p}$ value & HR & $95 \%$ CI & $\mathrm{p}$ value \\
\hline Gender & male & 0.98 & $0.60-1.58$ & 0.92 & 0.69 & $0.42-1.15$ & 0.15 \\
\hline Age & $>65$ & 1.76 & $1.14-2.70$ & 0.010 & 1.19 & $0.74-1.90$ & 0.47 \\
\hline $\mathrm{T}$ & 4 & 2.03 & $1.22-3.40$ & 0.0067 & 2.47 & $1.45-4.19$ & $<0.001$ \\
\hline $\mathrm{N}$ & positive & 0.96 & $0.60-1.53$ & 0.85 & 1.11 & $0.65-1.89$ & 0.70 \\
\hline G & high & 1.30 & $0.73-2.23$ & 0.37 & 0.82 & $0.42-1.60$ & 0.56 \\
\hline \multirow[t]{2}{*}{ Localization } & right & 1.25 & $0.73-2.13$ & 0.42 & 1.66 & $0.97-2.82$ & 0.06 \\
\hline & rectum & 1.05 & $0.62-1.78$ & 0.85 & 1.01 & $0.59-1.74$ & 0.97 \\
\hline KRAS & mut & 0.96 & $0.55-1.67$ & 0.88 & 0.98 & $0.54-1.78$ & 0.94 \\
\hline \multirow[t]{2}{*}{ Line } & 2nd & 1.15 & $0.63-2.10$ & 0.65 & 1.33 & $0.72-2.43$ & 0.36 \\
\hline & $>2 \mathrm{nd}$ & 3.34 & $1.28-8.71$ & 0.014 & 4.02 & $1.39-11.62$ & 0.010 \\
\hline \multirow[t]{3}{*}{ Backbone } & IRI doubl & 1.11 & $0.56-2.16$ & 0.77 & 1.25 & $0.56-2.79$ & 0.59 \\
\hline & OX doubl & 1.28 & $0.59-2.80$ & 0.53 & 0.89 & $0.36-2.21$ & 0.81 \\
\hline & other & 11.28 & $3.49-36.42$ & $<0.001$ & 2.51 & $0.72-8.74$ & 0.15 \\
\hline
\end{tabular}

$\mathrm{n}=$ Number; $\mathrm{HR}=$ hazard ratio; $\mathrm{p}=$ wald $\mathrm{p}$ value; mut = mutated; IRI = irinotecan; $\mathrm{OX}=$ oxaliplatin .

patients undergoing chemotherapy for metastasized CRC is rare. A retrospective study of 788 patients with metastasized CRC presented classification T3 or T4 and high tumor grade, and also presence of nodal disease $(\mathrm{N} 1 / \mathrm{N} 2)$, diffuse metastasis, high CEA levels and low Albumin levels to be significantly associated with adverse outcome after resection of the primary tumor [31]. In our series, lymph node status, tumor localization (right colon, left colon, rectum), and gender of the patients did not show an impact on the outcome. KRAS status could be obtained only from around $50 \%$ of the patients, most likely because KRAS status was not routinely tested in all patients when the majority of our cohort started treatment with bevacizumab. A prognostic impact on the outcome was not noted for KRAS status in our cohort. 
According to our findings, patients older than 65 years had significantly shorter PFS than younger patients following treatment with bevacizumab. The difference, however, accounted only for a few weeks and no difference in OS was observed. A decrease in OS of elderly patients receiving bevacizumab compared to younger patients has been reported by observational studies and subgroup analyses of clinical trials [32-35]. Slight reductions in both PFS and OS were observed in patients $\geq 70$ years compared to younger patients treated with first-line bevacizumab-combination regimens in a large German observational study [36]. However, a subgroup analysis from the AGTIG MAX trial found similar benefits of adding bevacizumab to capecitabine in elderly patients $>75$ years to those $<75$ years [37]. Furthermore, according to the prospective AVEX trial that specifically evaluated elderly patients, capecitabine and bevacizumab was an effective regimen for this group of patients [25]. According to a systematic review, benefits of bevacizumab in elderly patients do not appear to be significantly different from those reported in younger patients [34]. Therefore, it is questionable if the differences observed in the PFS between elderly and younger patients found in our analysis and previous studies are clinically relevant.

In addition to the 3 single center cohorts analyzed and presented in this study, a subgroup of patients enrolled in the CAIRO2 trial (randomized phase III study of capecitabine, oxaliplatin, bevacizumab with or without cetuximab in first-line advanced CRC) that underwent first-line combination chemotherapy with CapOX and bevacizumab (Arm A) at centers in the Netherlands has been included in the genomic analysis studies conducted within Angiopredict. The clinical characteristics of these patients have previously been described [18].

Data presented here comprised of 3 different retrospective cohorts collected by chart review. Nevertheless, the PFS for all 3 cohorts was similar, highlighting the reproducible benefits of a combination bevacizumab regimen even within heterogeneous cohorts. Data on OS differed, especially with respect to patients from the UHEI cohort, for whom OS was significantly longer. It is not possible to definitely ascertain the underlying reason for this difference in this retrospective study. A possible reason may be a higher proportion of patients undergoing (curative) resection of metastases or interventional therapies following bevacizumab administration within the UHEI cohort. When patients with resected metastasis were excluded from the UHEI cohort, the difference compared with other cohorts was reduced and no longer significant (data not shown). Data on resection of metas-

tases was not available for the other cohorts. Nevertheless, survival rates of up to 34 months have also been reported in other CRC studies and clinical trials $[28,30]$. To account for the possible cohort effect, all survival analyses (log-rank, Cox-regression) were performed with stratification by center.

Our analyses have further limitations. First, with respect to the retrospective nature of our study, we observed comparatively long survival rates in 2 nd and 3 rd line patients included in our analysis, which may be explained by selection bias. Also, ECOG status of our patients and adverse effects as a consequence of combination bevacizumab therapy were not analyzed in this study.

In conclusion, our data demonstrate that the survival of CRC patients receiving combination chemotherapy with bevacizumab was comparable to prospective clinical studies, providing evidence for demographically representative cohorts. A non-FP chemotherapy backbone was significantly associated with adverse outcome, while all standard combinations (doublets and single FP) resulted in comparable survival rates. T4 classification was associated with shorter PFS and OS, while age $>65$ years and high tumor grade were found to be associated with shorter PFS but not OS.

\section{Acknowledgements}

We thank Marion Hauke for excellent assistance with data collection. This work was financially supported by the Seventh Framework Programme (FP7) under contract No. 278981 (ANGIOPREDICT), ZOBEL (Zentrum für Geriatrische Onkologie und Biologie in der Metropolregion Rhein-Neckar) and the Dutch Cancer Society (KWF 2015-7882).

\section{Disclosure Statement}

R.D.H. received honoraria from Roche, Merck, Sanofi-Aventis, Amgen and support for Clinical trials from Roche, Merck, SanofiAventis, Amgen. T. G. received honoraria from Roche, Merck, AstraZeneca. All other authors declare that they have no conflict of interest.

References

Digestion 2016;94:129-137 DOI: $10.1159 / 000449412$
1 Torre LA, Bray F, Siegel RL, Ferlay J, LortetTieulent J, Jemal A: Global cancer statistics, 2012. CA Cancer J Clin 2015;65:87-108.

2 Midgley R, Kerr D: Colorectal cancer. Lancet 1999;353:391-399.

3 Curreri AR, Ansfield FJ, McIver FA, Waisman HA, Heidelberger C: Clinical studies with 5-fluorouracil. Cancer Res 1958;18:478484 . 
4 Poon MA, O’Connell MJ, Moertel CG, Wieand HS, Cullinan SA, Everson LK, et al: Biochemical modulation of fluorouracil: evidence of significant improvement of survival and quality of life in patients with advanced colorectal carcinoma. J Clin Oncol 1989;7: 1407-1418.

5 Ross PJ, Cunningham D: The management of colorectal cancer: chemotherapy. Crit Rev Oncol Hematol 1999;30:215-226.

6 de Gramont A, Figer A, Seymour M, Homerin M, Hmissi A, Cassidy J, et al: Leucovorin and fluorouracil with or without oxaliplatin as first-line treatment in advanced colorectal cancer. J Clin Oncol 2000;18:2938-2947.

7 Douillard JY, Cunningham D, Roth AD, Navarro $M$, James $R D$, Karasek $P$, et al: Irinotecan combined with fluorouracil compared with fluorouracil alone as first-line treatment for metastatic colorectal cancer: a multicentre randomised trial. Lancet 2000;355:10411047.

8 Prenen H, Vecchione L, Van Cutsem E: Role of targeted agents in metastatic colorectal cancer. Target Oncol 2013;8:83-96.

9 Cunningham D, Atkin W, Lenz HJ, Lynch HT, Minsky B, Nordlinger B, et al: Colorectal cancer. Lancet 2010;375:1030-1047.

10 Pavlidis ET, Pavlidis TE: Role of bevacizumab in colorectal cancer growth and its adverse effects: a review. World J Gastroenterol 2013; 19:5051-5060.

11 Hurwitz H, Fehrenbacher L, Novotny W, Cartwright T, Hainsworth J, Heim W, et al: Bevacizumab plus irinotecan, fluorouracil, and leucovorin for metastatic colorectal cancer. N Engl J Med 2004;350:2335-2342.

12 Macedo LT, da Costa Lima AB, Sasse AD: Addition of bevacizumab to first-line chemotherapy in advanced colorectal cancer: a systematic review and meta-analysis, with emphasis on chemotherapy subgroups. BMC Cancer 2012;12:89.

13 Saltz LB, Clarke S, Díaz-Rubio E, Scheithauer W, Figer A, Wong R, et al: Bevacizumab in combination with oxaliplatin-based chemotherapy as first-line therapy in metastatic colorectal cancer: a randomized phase III study. J Clin Oncol 2008;26:2013-2019.

14 Kabbinavar FF, Schulz J, McCleod M, Patel T, Hamm JT, Hecht JR, et al: Addition of bevacizumab to bolus fluorouracil and leucovorin in first-line metastatic colorectal cancer: results of a randomized phase II trial. J Clin Oncol 2005;23:3697-3705.

15 Tebbutt NC, Wilson K, Gebski VJ, Cummins MM, Zannino D, van Hazel GA, et al: Capecitabine, bevacizumab, and mitomycin in first-line treatment of metastatic colorectal cancer: results of the Australasian Gastrointestinal Trials Group Randomized Phase III MAX Study. J Clin Oncol 2010;28:3191-3198.
16 Kabbinavar F, Hurwitz HI, Fehrenbacher L, Meropol NJ, Novotny WF, Lieberman G, et al: Phase II, randomized trial comparing bevacizumab plus fluorouracil (FU)/leucovorin (LV) with FU/LV alone in patients with metastatic colorectal cancer. J Clin Oncol 2003;21: 60-65.

17 Giantonio BJ, Catalano PJ, Meropol NJ O'Dwyer PJ, Mitchell EP, Alberts SR, et al: Bevacizumab in combination with oxaliplatin, fluorouracil, and leucovorin (FOLFOX4) for previously treated metastatic colorectal cancer: results from the Eastern Cooperative Oncology Group Study E3200. J Clin Oncol 2007;25:1539-1544.

18 Tol J, Koopman M, Cats A, Rodenburg CJ, Creemers GJ, Schrama JG, et al: Chemotherapy, bevacizumab, and cetuximab in metastatic colorectal cancer. N Engl J Med 2009; 360:563-572.

19 Stathopoulos GP, Batziou C, Trafalis D, Koutantos J, Batzios S, Stathopoulos J, et al: Treatment of colorectal cancer with and without bevacizumab: a phase III study. Oncology 2010;78:376-381.

20 Potti A, Schilsky RL, Nevins JR: Refocusing the war on cancer: the critical role of personalized treatment. Sci Transl Med 2010;2: $28 \mathrm{~cm} 13$.

21 International Union Against Cancer U: TNM Classification of Malignant Tumours, ed 7. West-Sussex, Wiley-Blackwell, 2009.

22 Bosman FT, Carneiro F, Hruban RH, Theise ND: WHO Classification of Tumours of the Digestive System, ed 4. Lyon, IARC, 2010.

23 Harrell FE: Regression Modeling Strategies. Heidelberg, Springer, 2011.

24 Heinemann V, von Weikersthal LF, Decker T, Kiani A, Vehling-Kaiser U, Al-Batran SE, et al: FOLFIRI plus cetuximab versus FOLFIRI plus bevacizumab as first-line treatment for patients with metastatic colorectal cancer (FIRE-3): a randomised, open-label, phase 3 trial. Lancet Oncol 2014;15:1065-1075.

25 Cunningham D, Lang I, Marcuello E, Lorusso V, Ocvirk J, Shin DB, et al: Bevacizumab plus capecitabine versus capecitabine alone in elderly patients with previously untreated metastatic colorectal cancer (AVEX): an openlabel, randomised phase 3 trial. Lancet Oncol 2013;14:1077-1085.

26 Guan ZZ, Xu JM, Luo RC, Feng FY, Wang LW, Shen L, et al: Efficacy and safety of bevacizumab plus chemotherapy in Chinese patients with metastatic colorectal cancer: a randomized phase III ARTIST trial. Chin J Cancer 2011;30:682-689.

27 Passardi A, Nanni O, Tassinari D, Turci D, Cavanna L, Fontana A, et al: Effectiveness of bevacizumab added to standard chemotherapy in metastatic colorectal cancer: final results for first-line treatment from the ITACa randomized clinical trial. Ann Oncol 2015;26: 1201-1207.
28 Schwartzberg LS, Rivera F, Karthaus M, Fasola G, Canon JL, Hecht JR, et al: PEAK: a randomized, multicenter phase II study of panitumumab plus modified fluorouracil, leucovorin, and oxaliplatin (mFOLFOX6) or bevacizumab plus mFOLFOX6 in patients with previously untreated, unresectable, wildtype KRAS exon 2 metastatic colorectal cancer. J Clin Oncol 2014;32:2240-2247.

29 Strickler JH, Hurwitz HI: Bevacizumab-based therapies in the first-line treatment of metastatic colorectal cancer. Oncologist 2012;17: 513-524.

30 Kumachev A, Yan M, Berry S, Ko YJ, Martinez MC, Shah K, et al: A systematic review and network meta-analysis of biologic agents in the first line setting for advanced colorectal cancer. PLoS One 2015;10:e0140187.

31 Chew MH, Teo JY, Kabir T, Koh PK, Eu KW, Tang CL: Stage IV colorectal cancers: an analysis of factors predicting outcome and survival in 728 cases. J Gastrointest Surg 2012;16: 603-612.

32 Venderbosch S, Doornebal J, Teerenstra S, Lemmens W, Punt CJ, Koopman M: Outcome of first line systemic treatment in elderly compared to younger patients with metastatic colorectal cancer: a retrospective analysis of the CAIRO and CAIRO2 studies of the Dutch Colorectal Cancer Group (DCCG). Acta Oncol 2012;51:831-839.

33 Kozloff MF, Berlin J, Flynn PJ, Kabbinavar F, Ashby M, Dong W, et al: Clinical outcomes in elderly patients with metastatic colorectal cancer receiving bevacizumab and chemotherapy: results from the BRiTE observational cohort study. Oncology 2009;78:329-339.

34 Sclafani F, Cunningham D: Bevacizumab in elderly patients with metastatic colorectal cancer. J Geriatr Oncol 2013;5:78-88.

35 Rouyer M, Fourrier-Réglat A, Smith D, Becouarn Y, Guimbaud R, Tubiana-Mathieu N, et al: Effectiveness and safety of first-line bevacizumab plus FOLFIRI in elderly patients with metastatic colorectal cancer: results of the ETNA observational cohort. J Geriatr Oncol 2016;7:187-194.

36 Hofheinz R, Petersen V, Kindler M, Schulze M, Seraphin J, Hoeffkes HG, et al: Bevacizum$\mathrm{ab}$ in first-line treatment of elderly patients with metastatic colorectal cancer: German community-based observational cohort study results. BMC Cancer 2014;14:761.

37 Price TJ, Zannino D, Wilson K, Simes RJ, Cassidy J, van Hazel GA, et al: Bevacizumab is equally effective and no more toxic in elderly patients with advanced colorectal cancer: a subgroup analysis from the AGITG MAX trial: an international randomised controlled trial of Capecitabine, Bevacizumab and Mitomycin C. Ann Oncol 2012;23:1531-1536. 